\title{
AN INTELLIGENT BASED SYSTEM FOR BLIND PEOPLE MONITORING IN A SMART HOME
}

\author{
Pamely Zantou ${ }^{1}$, Mikael A. Mousse ${ }^{2}$, Béthel C. A. R. K Atohoun ${ }^{3}$ \\ ${ }^{1}$ Laboratoire de Recherche en Sciences Informatiques et Applications \\ Institut de Formation et de Recherche en Informatique \\ Université d'Abomey-Calavi, Benin \\ ${ }^{2}$ Institut Universitaire de Technologie \\ Université de Parakou, Benin \\ ${ }^{3}$ Ecole Supérieure de Gestion d'Informatique et des Sciences
}

\begin{abstract}
Visually impaired people need help to travel safely. To make this possible, many travel aids have been designed. Among them, the cane which is considered as a symbol of visual deficiency in the whole world. In this work, we build an electronic white cane using sensors' technology. This intelligent cane detects obstacles within $2 m$ on the ground or in height, and sends vocal instructions via a Bluetooth headset. We have also built a mobile application to track in real time the visually impaired and a WEB application to control the access to the mobile one. We use ultrasound, IR sensors and a raspberry pi to process data. We use Python as programming language for electronic devices. The mobile application is Android. Though, the WEB application is a REST API developed using Python and Java Script.
\end{abstract}

\section{KEYWORDS}

Electronic white cane; Sensors; human monitoring; smart home.

\section{INTRODUCTION}

Many situations can deprive human beings from freedom. Visual impairment is one that make people completely dependent on their caregivers. Indeed, a visually impaired person cannot move freely or carry out any activity that requires vision. To facilitate their integration into society and allow them to make good decisions while moving, they benefit from external assistance provided by caregivers, trained dogs, white canes, small electronic devices. The white cane remains one of the most widely used tools by visually impaired. It not only enables visually impaired people to avoid obstacles. This device is widely recognized as symbol of blindness. However, it does not allow the visually impaired to freely carry out their daily activities. It does not detect obstacles above the belt such as: truck mirrors, sloped branches, advertising signs. It is also not possible to be aware of the presence of a close obstacle before touching or detecting it. These different situations expose visually impaired people to frequent severe body shocks. To avoid this situation, electronic canes have been invented. They use sensors or stereovision technologies. Tremendous efforts are made today to improve electronic travel aid solutions. Unfortunately, these solutions are almost unused or unknown in most African countries. Over $90 \%$ of people suffering from visual impairment live in developing countries. Africa is the most vulnerable continent of all. Moreover, 
the solutions on the market are not suited to our realities. This paper aims to produce an electronic cane model, easy to use, and suited to Africa's realities.

This paper is organized as follows. Section 2 presents related work to see the different approaches for assisting the displacement of blind people. Section 3 presents details of our solution. We present the system overview, sensors choice and configuration. Finally, Section present results and discussion.

\section{STATE OF THE ART}

Navigation systems for blind people require storing and retrieving information for path planning, generating directions, and providing location information. Depending on the approach used, this information may include floor plans, the location and description of objects in the indoor environment, locations of identifier tags or data collected by sensors in the environment. Based on studies about visually impaired needs, the design of an electronic smart cane seems to be the best way to help them in their daily life. It is globally recognized as a symbol of visual deficiency. It makes the blind feel safe. It is a handy tool that can be easily used by anyone, even disabled people. To overcome problems associated with traditional white canes, Electronic Travel Aids, ETAs, have been invented since the $19^{\text {th }}$ century. In [1], the author defines ETAs as active devices that emit and receive waves (electromagnetic or acoustic) to explore the environment within a certain perimeter, or passive receivers that receive the light reflected by the different obstacles. They process the information and provide the user with information on the configuration of nearby obstacles. Electronic canes identify physical quantities and transform them into information thanks to the knowledge of the physical phenomena involved. They provide information on the shape of obstacles, their dimensions, colors and even the distance between them and the user.

Dhruv Jain et al. developed a system Roshni [12], that can be used for indoor navigation system for blind person. This system consists of the following functional components: assistance for determining the user's position in a building, a detailed interior map of the building and a mobile application. By pressing keys on the mobile unit, directions concerning position, orientation and navigation can be obtained from the portable system via acoustic messages. A RFID based navigation system proposed by Punit Dharani et al. [13]. The system provides a technological solution for the visually impaired to travel through public locations easily using RFID. Parth Mehta et al. proposed a novel indoor navigation system for visually impaired people [14] and the paper illustrates a structure which uses the IR sensor and magnetic compass on the VI-Navi handheld device to determine the location and orientation of the user in a fast and a robust manner using a voice enabled GPS inside a closed environment.

ETAs' industry, has been undergoing a constant and remarkable evolution for several years now. Thus, many generations of solutions have been developed. These ETAs can be classified in terms of technologies used to build them. We can identify two main generations of electronic canes. Electronic canes using:

- ultrasound sensors and/or laser and/or IR sensors [2, 4, 5];

- cameras, electro-tactile system, Oh I see (the vOICe), stereovision systems [6-8].

Depending on these different technological approaches, Table 1 presents some existing ETAs. 
Table 1. Listing of some existing solutions.

\begin{tabular}{|c|c|c|c|c|}
\hline ETAs & $\begin{array}{c}\text { Technological } \\
\text { approach/principle }\end{array}$ & Manufacturer & $\begin{array}{c}\text { Year of } \\
\text { invention }\end{array}$ & $\begin{array}{c}\text { Type of } \\
\text { Alert }\end{array}$ \\
\hline SmartCane & Ultrasound & $\begin{array}{c}\text { Indian Institue } \\
\text { of Technology, } \\
\text { New delhy }\end{array}$ & 2007 & Vibrations \\
\hline UltraCane & Ultrasound & $\begin{array}{c}\text { Sound Foresight } \\
\text { Technology } \\
\text { Limited }\end{array}$ & 2011 & Vibrations \\
\hline TOM & IR & $\begin{array}{c}\text { Foundation } \\
\text { vision }\end{array}$ & 2013 & Vibrations \\
\hline WeWalk & Ultrasound & $\begin{array}{c}\text { Young Guru } \\
\text { Academy }\end{array}$ & 2016 & $\begin{array}{c}\text { Vocal } \\
\text { instructions }\end{array}$ \\
\hline Sherpa & Stereo Vision & HANDISCO & 2017 & $\begin{array}{c}\text { Vocal } \\
\text { instructions }\end{array}$ \\
\hline
\end{tabular}

\section{Proposed System}

The proposed system is a set of a smart stick and a real time tracking mobile application. The smart stick uses a network of ultrasound and IR sensors to detect obstacles (within a range of $2 \mathrm{~m}$ in height or on the ground), a Raspberry Pi to process data and a GPS module to collect satellite data. On the one hand, sensors send data collected from the blind's environment to the Raspberry Pi. This last, processes the data and send vocal instructions to the visually impaired through a Bluetooth headset connected to the smart stick. On the other hand, the GPS module forwards satellite information to the Raspberry Pi which processes them. Then, sends them back to mobile and WEB applications via a REST API.

\subsection{System Overview}

The proposed system is presented by Fig. 1 .

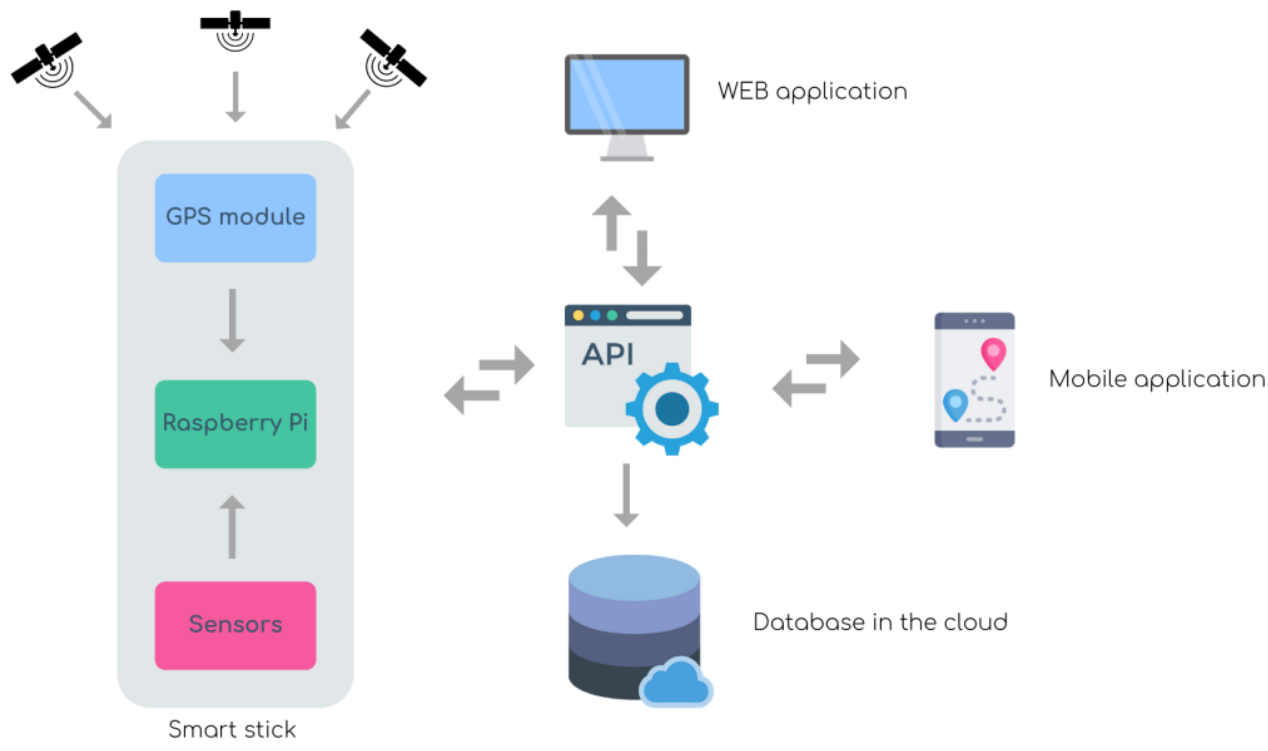

Figure $1:$ System architecture 
The system has three major modules: smart stick, web application and mobile Application. These modules communicate through an API. The intelligent cane is a normal white which embeds sensors. A sensor is a device that transforms a physical quantity (temperature, pressure, concentration, pressure) into a usable quantity which is generally electrical. There is a large number of sensors that differ from the physical quantity they measure. Among them, we have: ultrasound, temperature, pressure, acceleration, infrared, laser, radar sensors. As far as the proposed system is concerned, sensors enable us to evaluate the distance between the visually impaired and obstacles. The most widely used distance sensors are:

- Ultrasound sensors;

- Laser sensors;

- IR sensors;

- Radar sensors;

\subsection{Choice of sensors}

As far as the proposed system is concerned, sensors enable us to evaluate the distance between the visually impaired and obstacles. The most widely used distance sensors are: ultrasound sensors, laser sensors, IR sensors, radar sensors. Some Criteria are used to choose sensors. They are range, precision, cost and interference sensitivity. The laser and radar sensors can detect near and far obstacles. However, laser sensors are very expensive, which contradicts our aim to provide people with an affordable solution. They are also less precise for close obstacles detection. Radar sensors are not able to detect small objects because of their medium accuracy, which is not good for our system. Ultrasound sensors detect close obstacles with a very good accuracy depending on their measurement cone. Nevertheless, very close obstacles are less likely to be detected. IR sensors are very accurate but only detects very close obstacles. Both, ultrasound and IR sensors are cheap and detect small objects. They fit with our system requirements. Actually, we use an ultrasound sensor HC-SR04 and an IR sensor Sharp GP2Y0A02YK0F for the system.

\section{1) GPS module NEO-6M}

The GPS module provides location data (longitude and latitude) from satellites. This is collected with the GPS module EEPROM antenna. It sends a big stream of data in NMEA format. NMEA consists in several sentences. However, we just need one sentence. This sentence starts with \$GPGGA. It contains useful information such as: coordinates, time. These information are comma separated. Thus, we can extract information from \$GPGGA string by counting the commas. The latitude is found after two commas and the longitude after four commas. The GPS module sends data collected to the cane's process unit which is the Raspberry Pi.

\section{2) Raspberry Pi}

To make the system works, we need a Logical Processing Unit (LPU)to communicate sensors' data to the stick's users. For this purpose, we can use microcontrollers such as Arduino, PIC18F46k80, etc. However, we are dealing with a two modules system. In fact, we have the stick and a software linked to it. A real computer is the best candidate to make them talk together. Then, we use the Raspberry Pi Zero W (Fig. 6) as the stick's LPU. It is a monocard nano computer with a single core $1 \mathrm{GHz}$ processor, a micro SD card, a mini HDMI port, two micro USB ports, (one for power, one for USB), and 512MB of RAM. It has a built-in WiFi and bluetooth. It needs $5 \mathrm{~V}$ as operating voltage. The Raspberry Pi Zero W processes data collected by 
sensors and send them either to the REST API or the users. In fact, it computes the distance between the user and the obstacle and sends vocal instructions to users via a Bluetooth headset.

The Raspberry Pi Zero W only deals with digital signals. Ultrasound sensors and the GPS module send digital signals, directly processed by the Pi. However, IR sensors send analog signals. Our system uses an Analog to Digital Converter (ADC) to transform analog information into digital one. The IR sensor sends an analog signal in the form of voltage. If the voltage sent is not $0 \mathrm{~V}$, then, an obstacle is detected, and the ADC communicates this state to the Raspberry Pi as numeric value. The Raspberry Pi Zero W has two built-in Universal Asynchronous ReceiverTransmitter (UARTs), a PL011 and a mini UART. By default, on the Raspberry Pi Zero W, PL011 UART is connected to the Bluetooth module while the mini UART is used as the primary UART and will have a linux console on it. Because of the amount of the GPS module data stream, we recongure the Pi. We connect the bluetooth module with the mini UART and the primary UART to the Linux console. This new configuration helps us recover GPS data and process it on the $\mathrm{Pi}$. The Raspberry PiWis also responsible of voice synthesis. In fact, it holds a text-to-speech engine which enables us to send vocal instructions to the stick's users. We use SVOX Pico, a small-footprint text-to-speech engine distributed with the Android operating system, but it can also be run on Linux and other POSIX systems. Since, Raspberry Pi runs on raspbian, a debian based OS, SVOX Pico is an optimal choice.

\section{3) IR sensor Sharp GP2Y0A02YK0F}

The IR sensor chosen is the Sharp GP2Y0A02YK0F. It is composed of an integrated combination of PSD (Position Sensitive Detector), IRED (Infrared Emitting Diode) and a signal processing circuit. The variety of the reflectivity of the object, the environmental temperature and the operating duration are not influenced easily to the distance detection because of adopting the triangulation method. Its distance measuring range is $20 \mathrm{~cm}$ to $150 \mathrm{~cm}$. This device outputs an analog voltage corresponding to the detection distance. It needs 4.5 to $5.5 \mathrm{~V}$ supply voltage to work.

\section{4) Ultrasound sensor HC-SR04}

The ultrasound sensor chosen for the stick is the HC-SR04. Its detection range is $2 \mathrm{~cm}$ to $400 \mathrm{~cm}$ with $3 \mathrm{~mm}$ of precision, $40 \mathrm{kHz}$ as frequency, $5 \mathrm{~V}$ for the operating voltage, $5 \mathrm{~V}$ as digital output and $30^{\circ}$ as detection angle. Ultrasonic sensors use a transducer to send and receive ultrasonic pulses that relay back information about an object's proximity. Indeed, the traducer receives distinct echo patterns. They can measure distances quite accurately $[18,4,12]$. The sensor determines the distance to a target by measuring time lapses between the sending and receiving of the ultrasonic pulse.

\section{5) REST API}

The smart cane is the blind's decision-making tool. To help the blind's parent track him/her in real time, it is necessary to link the cane to a mobile application. To make the mobile app talk with the cane, we use an Application Programming Interface (API). The API is a Representational State Transfer (REST) one. In fact, it is a standard invented by Roy Fielding in his dissertation [11]. REST APIs are based on the Hypertext Transfer Protocol (HTTP) and mimic the way the WEB works in client-server communications. The client-server principle involves two entities that interact in a REST API : the cloud database and application modules which are clients. To interact with the cloud-based database, applications send HTTP requests: GET, POST, PUT or DELETE to the API. This last query the database. Requests' responses are still sent to clients through the API. 


\subsection{Electronic Configuration}

In order to detect obstacles accurately, sensors should be placed at optimal positions on the stick (Fig. 2). We propose a cane of length 1 , depending on its user height. The angle $\beta$, between the cane and the horizontal which is an input parameter. The proposed system uses three sensors. Two ultrasound sensors and an IR sensor. The IR sensor will detect obstacles on the ground and at a distance $\mathrm{d} 1$ less than or equal to $1 \mathrm{~m}(\mathrm{~d} 1<=1 \mathrm{~m})$. The lower ultrasound sensor, on the one hand, is placed at distance $\mathrm{d} 4$ and will detect obstacles on the ground at a distance $\mathrm{d}$ between $\mathrm{d} 1=$ $1 \mathrm{~m}$ and $\mathrm{d} 2=2 \mathrm{~m}(\mathrm{~d} 1<=\mathrm{d}<=\mathrm{d} 2)$. The upper ultrasound sensor, on the other hand, can detect obstacles above the belt up to $2 \mathrm{~m}$ in height.

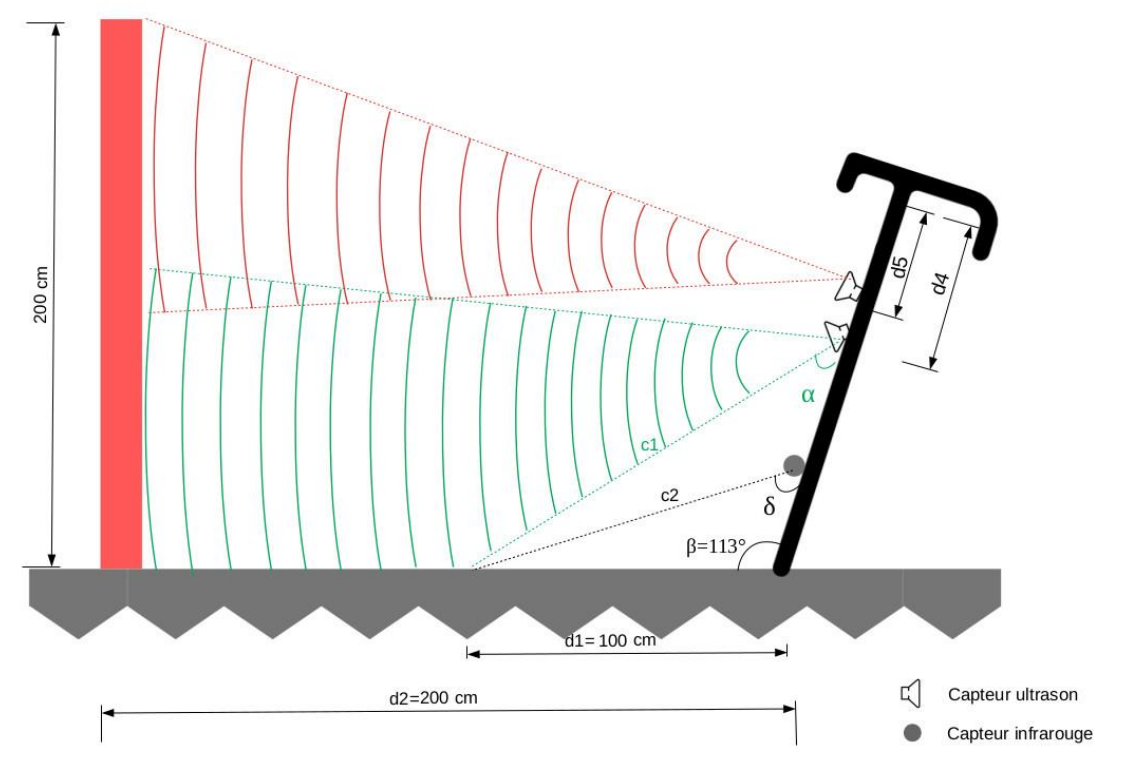

Figure 2: Electronic configuration

Several parameters are involved in the cane's electronic configuration. We will be interesting in finding $\gamma, \alpha, \delta, \mathrm{c} 1$ and $\mathrm{c} 2$ (Fig. 3).

- $\gamma$ : the angle between the cane and the upper ultrasound sensor;

- $\alpha$ : the angle between the cane and the lower ultrasound sensor;

- $\delta$ : the angle between the IR sensor and the cane;

- $\quad \mathrm{c} 1$ : the distance between the end of the ultrasound sensor and the floor;

- $\quad \mathrm{c} 2$ : the distance from the end of the IR sensor to the floor.

To calculate them, we used geometric properties including the theorem of Al-Khashi or the law of cosines. 


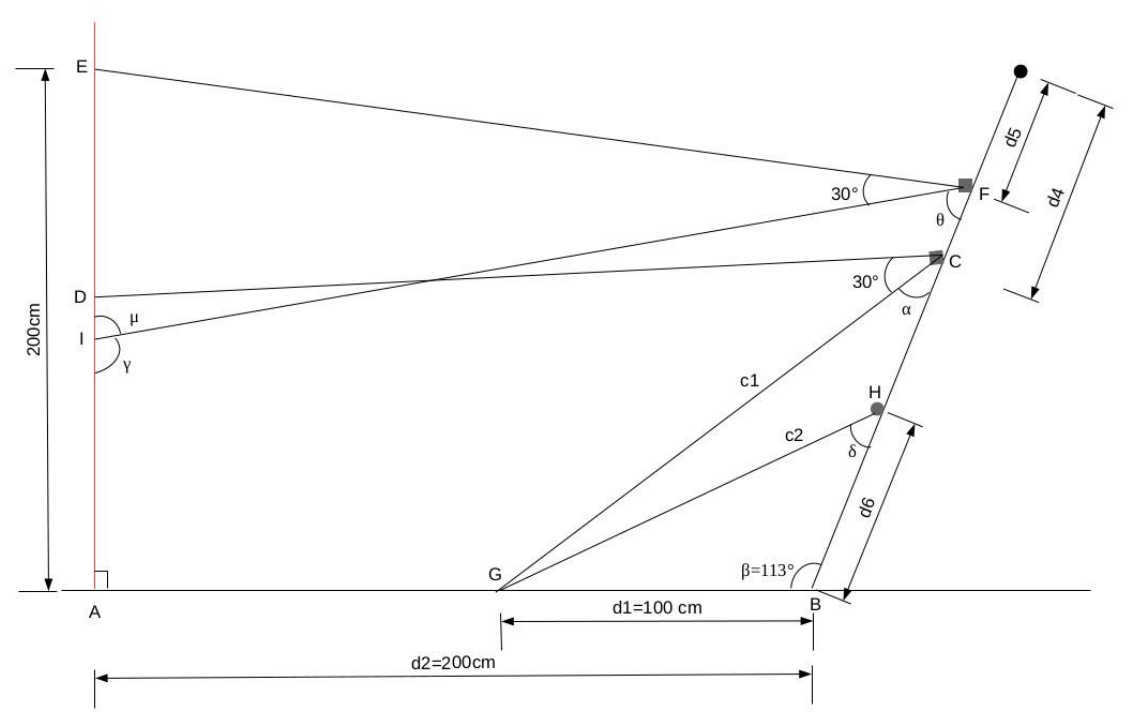

Figure 3: Electronic configuration: plan view

The table 1 summarizes the sensors' inclination angles information.

Table 2. Sensors' inclination angles

\begin{tabular}{|c|c|}
\hline Sensors & Measuring the angle of inclination \\
\hline Upper ultrasound sensor & $52^{\circ}$ \\
\hline Lower ultrasound sensor & $46^{\circ}$ \\
\hline IR sensor & $40^{\circ}$ \\
\hline
\end{tabular}

\subsection{System driven circuit}

In this section, we are going to present the electrical circuit that controls the system. The circuit shows us how the different electronic components are mounted to operate in the system. In addition, to the components mentioned in the previous section, we have, push buttons to activate the cane or alert in case of emergency, resistors, a buzzer for audible beeps and a Light Emitting Diode (LED). The components of the circuit are connected to the inputs of the Raspberry Pi (pins) including the ground, GND and VCC which provides an input voltage of $5 \mathrm{~V}$. The whole system relies on a breadboard (Fig. 4). 


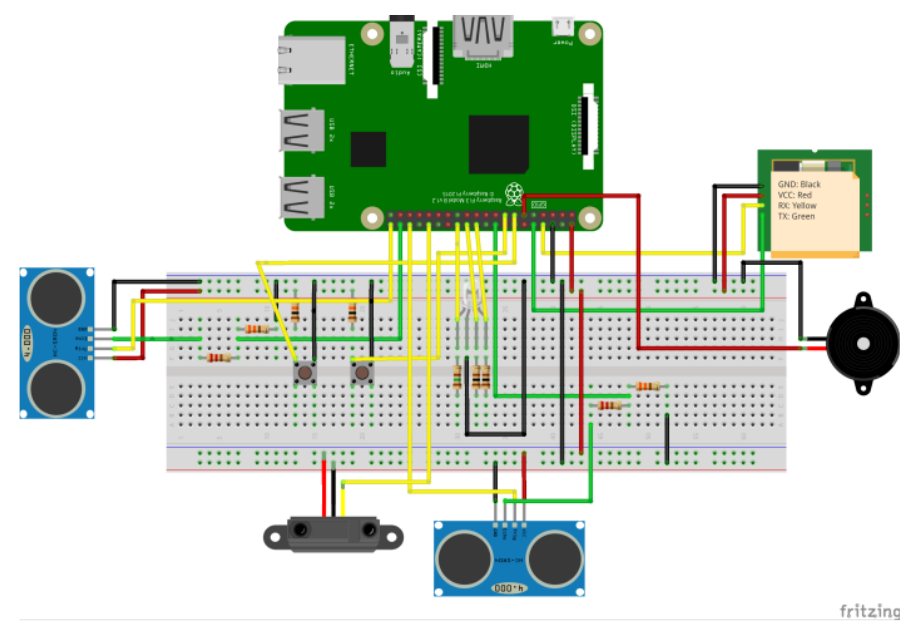

Figure 4: System driven circuit

\section{RESULTS AND DISCUSSION}

Before assembling all the cane's components, we test each of them. Based on the time used by the ultrasound sensor to reach obstacles and analog outputs of the IR sensor we can evaluate our system performances. The table 2 summarizes results derived from computations and measurements. Even though the proposed solution has been influenced by [3]. The results show that combining infrared range and ultrasonic sensors may lead to a better decision-making system. The proposed system is also connected to mobile application to track the visually impaired people in real time.

Table 3. Comparison of computed and measured results

\begin{tabular}{|c|c|c|c|c|c|c|}
\hline & \multicolumn{3}{|c|}{ Ultrasonic sensor } & \multicolumn{3}{c|}{ IR Sensor } \\
\hline $\begin{array}{c}\text { Distance } \\
(\mathrm{cm})\end{array}$ & $\begin{array}{c}\text { Time } \\
\text { computed } \\
(\mathrm{s})\end{array}$ & $\begin{array}{c}\text { Time } \\
\text { measured (s) }\end{array}$ & Error(s) & $\begin{array}{c}\text { Analog } \\
\text { output } \\
\text { computed } \\
(\mathrm{V})\end{array}$ & $\begin{array}{c}\text { Analog } \\
\text { output } \\
\text { measured } \\
(\mathrm{V})\end{array}$ & Error (V) \\
\hline 50 & 0.29 & 0.3 & 0.01 & 1.3 & 1.3 & 0 \\
\hline 75 & 0.44 & 0.45 & 0.01 & 0.8 & 0.7 & 0.01 \\
\hline 100 & 0.59 & 0.62 & 0.03 & 0.6 & 0.59 & 0.01 \\
\hline 125 & 0.74 & 0.78 & 0.04 & 0.52 & 0.51 & 0.01 \\
\hline 150 & 0.88 & 0.92 & 0.04 & 0.5 & 0.485 & 0.015 \\
\hline 175 & 1.03 & 1.07 & 0.04 & 0.3 & 0.282 & 0.018 \\
\hline 200 & 1.18 & 1.23 & 0.05 & 0.2 & 0.09 & 0.11 \\
\hline
\end{tabular}

The system prototype is the set of the physical cane, the REST API, the Android application and the WEB application.

\subsection{Cane}

The is a set of the sensors, the raspberry pi, the buzzer, a battery and a USB input to charge the battery. Fig. 5 presents the prototype of the white cane. 


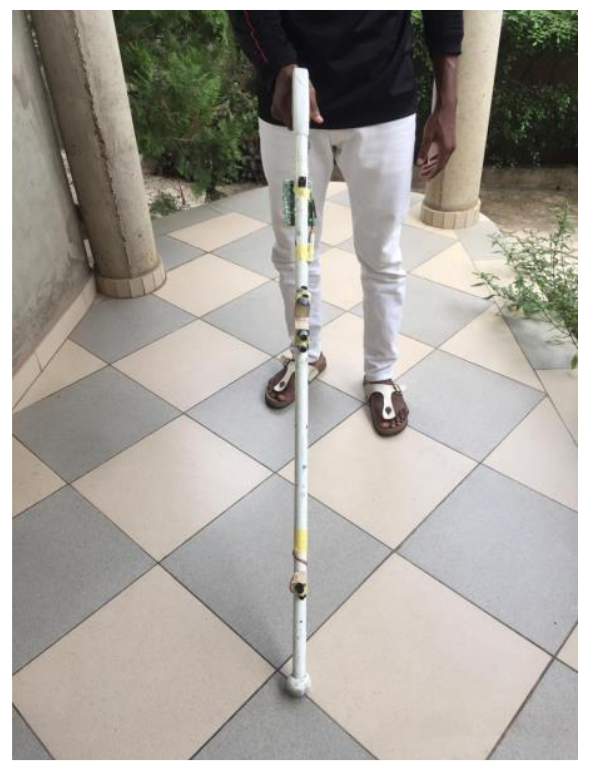

Figure 5: Cane prototype

\subsection{The API}

The API is a python module that contains classes which methods are HTTP requests: get(), post(), put() and delete(). It allows you to authenticate from the Android application, send geolocation data (from the GPS module) to the mobile application.

\subsection{WEB Application}

The WEB application is a dashboard (see Fig. 6). To access it, you must first authenticate yourself. Once the authentication is successful, the home page is displayed. The site has five rubrics. They are:

- Home: There is information about each rubric;

- Add parent: This is a form to add a parent to the database;

- Parents list: This form is used to check the list of Parents which are store in the system;

- Visually impaired: This page contains the information about the visual impaired, a map on which he can be geolocated, a panel to contact a relative and a button to add a visual impaired;

- User guide: The system user guide. 


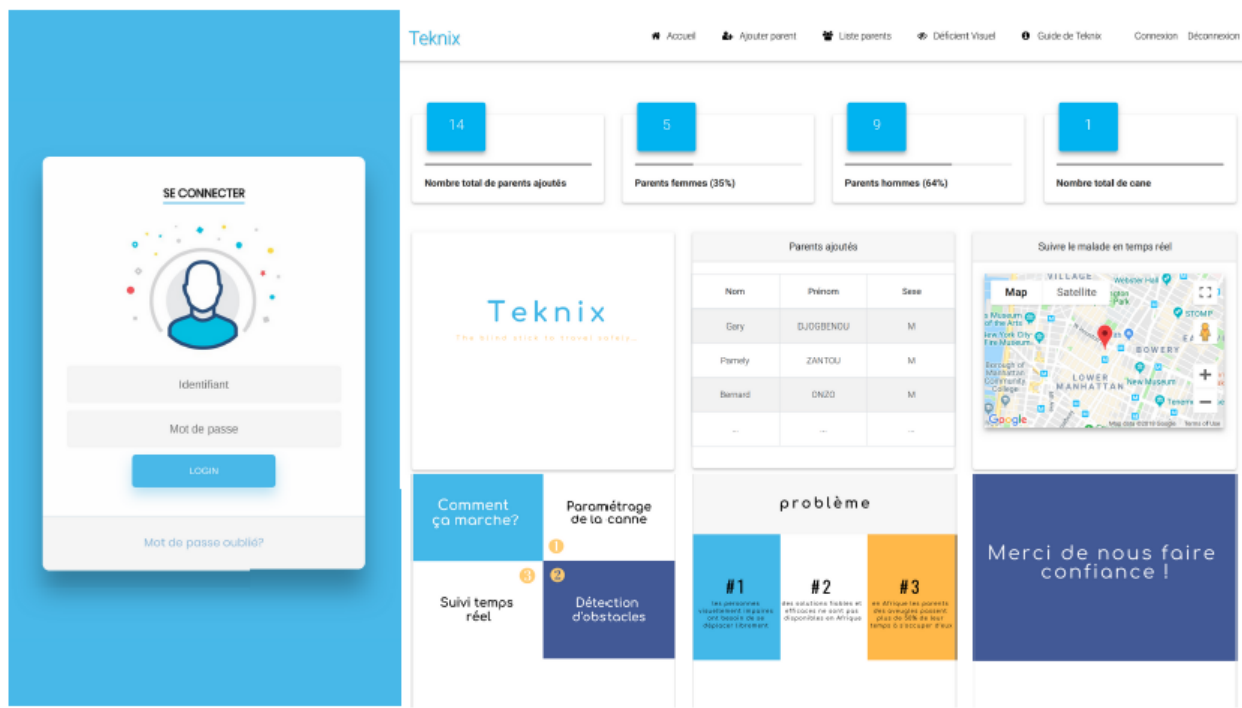

Figure 6: WEB application

\subsection{Mobile application}

The Android application allows you to track the visually impaired in real time. To have access to these features, you must first authenticate yourself. Once authentication is successfully completed, the user can track the visual impaired. The Android application's authentication interface is presented by Fig. 7 .

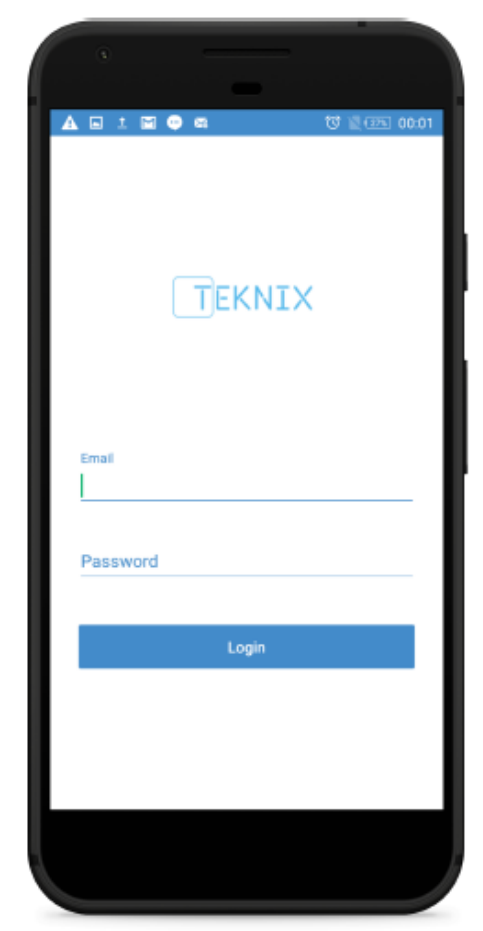

Figure 7: Android application's authentication interface.

In addition, voice commands transmission via the headset is automatic. Because of this, we made a compromise related to the Universal Transmitter/Receiver (UART) of the raspberry pi. The 
raspberry pi has two UARTs: a PL011 or UART0 connected to the Bluetooth module and a miniUART connected to the Linux console. For reasons of reliability and data flow sent by the GPS module, we reconfigured the UART of the raspberry pi. The UART0 has been connected to the Linux console and the mini-UART connected to the Bluetooth module. Thus, the performance of the Bluetooth module decreases since the mini-UART is less powerful than the UART0.

After the implementation of the system and we compare the results to the state-of-the-art result. The results are mentioned in Table 3.

Table 3. Performance Evaluation

\begin{tabular}{|c|c|c|c|c|c|}
\hline Devices & Detection Range & $\begin{array}{c}\text { Time } \\
\text { Response }\end{array}$ & $\begin{array}{c}\text { Power } \\
\text { Consumption }\end{array}$ & $\begin{array}{c}\text { Stair } \\
\text { detection }\end{array}$ & $\begin{array}{c}\text { Tracking } \\
\text { module }\end{array}$ \\
\hline Proposed system & Medium & Fast & Low & Yes & Yes \\
\hline $\begin{array}{c}\text { New electronic white } \\
\text { cane for stair case } \\
\text { detection and } \\
\text { recognition using } \\
\text { ultrasonic } \\
\text { sensor }\end{array}$ & Medium & Fast & Low & Yes & No \\
\hline $\begin{array}{c}\text { New electronic white } \\
\text { cane for stair case } \\
\text { detection and } \\
\text { recognition using } \\
\text { ultrasonic } \\
\text { sensor }\end{array}$ & High & Fast & Low & Yes & No \\
\hline $\begin{array}{c}\text { Ultrasonic Spectacles } \\
\text { and Waist-belt for } \\
\text { Visually Impaired } \\
\text { and Blind Person }\end{array}$ & High & Fast & Low & No & No \\
\hline
\end{tabular}

The results mentioned in table 3 prove the efficacity of our propose method. In addition to detecting obstacles, our system makes it possible to follow the person over time

\section{CONClusion}

Visually impaired people represent $5 \%$ of the world's population. More than 26 millions of them are in Africa. They need social integration like any able-bodied person. This work presents an electronic cane that enables visually impaired to move around without having to resort to caregivers or traditional solutions. Indeed, it helps its user to detect obstacles in height as well as on the ground within a radius of two metres $(2 \mathrm{~m})$, which sends voice commands back to its user to enable him to avoid the obstacle that stands in his path and which has applications to follow him. The proposed cane has limitations that have been raised in the work. This allows us to consider interesting perspectives to improve our work. A camera module and a training could be used to make the cane a more intelligent assistant. This assistant will be able to accurately say how the patient could avoid an obstacle. The implementation of the retrace route function would make the Android application much more useful to the parents of the visual patient.

\section{REFERENCES}

[1] J. Villanueva: "Contribution a la télémetrie optique active pour l'aide aux déplacements des nonvoyants", Université Paris Sud - Paris XI

[2] T. Terlau and W. M. Penrod, "K'Sonar Curriculum Handbook", Available from: "http://www.aph.org/manuals/ksonar.pdf", June 2008 
[3] S. A. Bouhamed and I. K. Kallel and D. S. Masmoudi, "New electronic white cane for stair case detection and recognition using ultrasonic sensor", 2013, (IJACSA) International Journal of Advanced Computer Science and Applications

[4] L. Whitney, "Smart cane to help blind navigate", Available from: "http://news.cnet.com/830117938_105-10302499-1.html", 2009.

[5] J.M. Hans du Buf, J.Barroso, Jojo M.F. Rodrigues, H.Paredes, M.Farrajota, H.Fernandes, J.Jos, V.Teixeira, M.Saleiro."The SmartVision Navigation Prototype for Blind Users". International Journal of Digital Content Technology and its Applications, Vol.5 No.5, pp. 351-361, May 2011.

[6] P. Meijer, "An Experimental System for Auditory Image Representations". IEEE Transactions on Biomedical Engineering, vol.39, no 2, pp. 112-121, Feb 1992.

[7] M. Nie, J. Ren, Z. Li et al., "SoundView: an auditory guidance system based on environment understanding for the visually impaired people". in Proceedings of the 31st Annual International Conference of the IEEE Engineering in Medicine and Biology Society: Engineering the Future of Biomedicine (EMBC â09), pp.7240â7243, IEEE, September 2009.

[8] G. Balakrishnan, G. Sainarayanan, R. Nagarajan and S. Yaacob. "Wearable Real-Time Stereo Vision for the Visually Impaired". Engineering Letters, vol. 14, no. 2, 2007.

[9] B.Hoyle, D.Withington, D.Waters, "UltraCane", Available from: "http://www.soundforesight.co.uk/index.html". June 2006.

[10] Amit kumar, M. Manjunatha and J. Mukhopadhyay, "An Electronic Travel Aid for Navigation of Visually Impaired Person". Proceeding of the 3rd International Conference on Communication Systems and Networks, pp.1-5, 2011.

[11] Roy Fielding, "Architectural Styles and the Design of Network-based Software Architectures, PhD dissertation, 2000.

[12] D.Jain and M.Balakrishnan, P.V.M.Rao., " Roshni: Indoor Navigation System for Visually Impaired"

[13] P.Dharani, B.Lipson and D.Thomas, "RFID Navigation System for the Visually Impaired", Worcester Polytechnic Institute, 2012.

[14] P.Shah, P.Mehta, P.Kant and A.K.Roy, "VI-Navi: A Novel Indoor Navigation System for Visually Impaired People"

\section{AUTHORS}

Pamely Zantou received his B. Sc. in Informatics From Université d'Abomey-Calavi in 2018. Currently he is pursuing his master's degree in Artificial Intelligence. He is interested in unsupervised learning approaches for human activity recognition.

Mikael A. Mousse is an assistant professor in computer science at the Institut Universitaire de Technologie of Université de Parakou, Benin. His current research concerns about the automatic visual surveillance of wide area scenes using computational vision. His research interests focus on the design of multi-camera system for real-time multi-object tracking and human action recognition. He is recently focusing on the uncertainty management over the vision system using graphical models, and beliefs propagation. He is also interested in unsupervised learning approaches for human activity recognition.

Béthel Atohoun obtained his University Diploma in Scientific Studies (DUES 2) from the University of Abomey Calavi in 1993. He obtained his Masters in Computer Engineering in 1999 from the African Institute of Computer Science. He spent 6 years in a corporate environment as a computer engineer from 2000 to 2005. he proceeded to private higher education in October 2005 at ECOLE SUPÉRIEURE DE GESTION D'INFORMATIQUE ET DES SCIENCES (ESGIS). He held the position of lecturer and Head of IT, Networks and Telecommunications Department from 2007 to 2013. He obtained his PhD in Computer Science in 2013 from the Université du Littoral Côte d'Opale (ULCO). Since 2014, he has been the Director of Studies at ESGIS. His research interests include computer vision, multi-camera detection and tracking, theory of evidence, multi-agent systems and artificial intelligence.

(C) 2020 By AIRCC Publishing Corporation. This article is published under the Creative Commons Attribution (CC BY) license. 